Supplement of Biogeosciences, 14, 5313-5321, 2017

https://doi.org/10.5194/bg-14-5313-2017-supplement

(C) Author(s) 2017. This work is distributed under

the Creative Commons Attribution 3.0 License.

(c) (1)

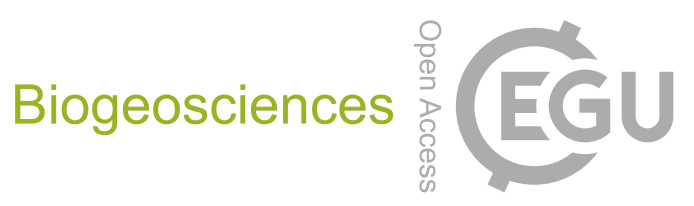

Supplement of

\title{
Parallel functional and stoichiometric trait shifts in South American and African forest communities with elevation
}

\section{Bauters et al.}

Correspondence to: Marijn Bauters (marijn.bauters@ugent.be)

The copyright of individual parts of the supplement might differ from the CC BY 3.0 License. 

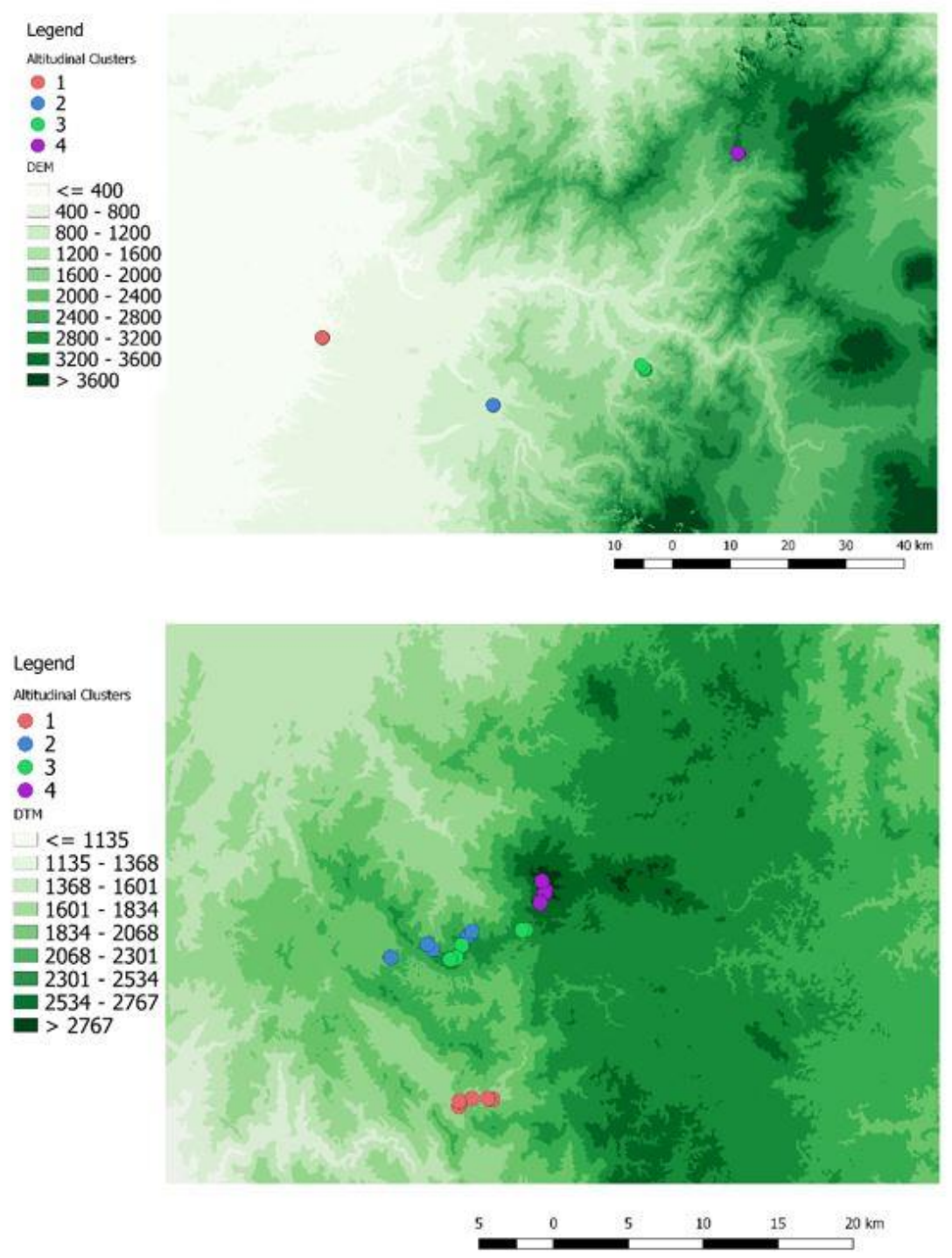

$4 \quad$ Fig. S1.

5 Overview map with the locations of the Ecuador transect (upper; 400-3200 masl, 4-5 plots per

6 cluster) and the Rwanda transect (lower; 1600 - 3000 masl, 5 plots per cluster) plot locations

7 projected on a DEM (based on the Aster GDEM product (1). For clarity different scale legends $8 \quad$ are used in both maps. 

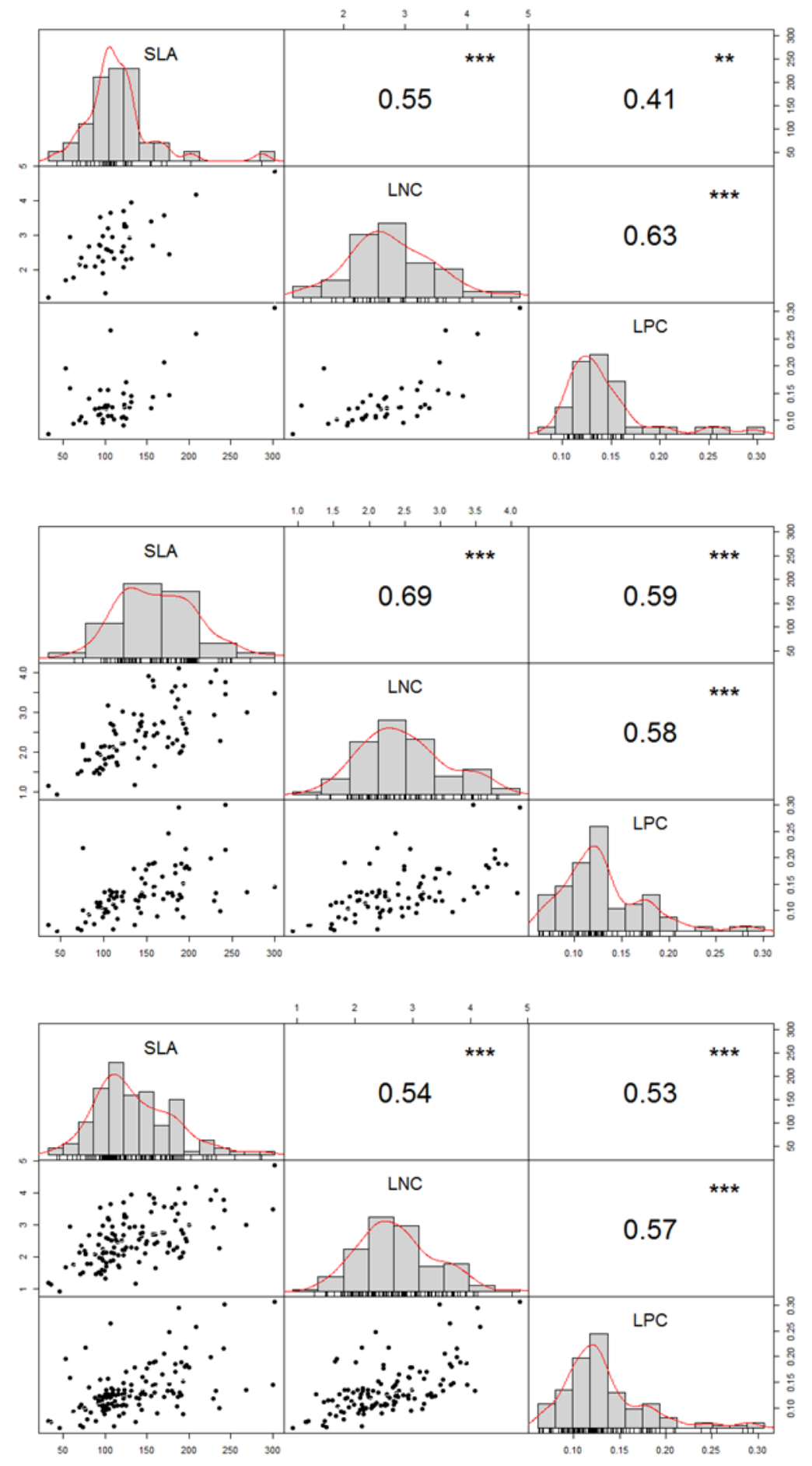

Fig. S2.

11 Structure and correlations of the trait data in the Rwanda (upper), Ecuador (middle) and both 12 (lower) transects with Spearman correlation statistics and their significances (p-value $<0.001 * * *$, 
$13<0.01 * *,<0.05^{*},<0.1$.). Diagonal shows the probability density function as fitted with fitted 14 kernel density plots. Shown for specific leaf area (SLA), leaf nitrogen content (LNC), and leaf 15 phosphorus content (LPC). 

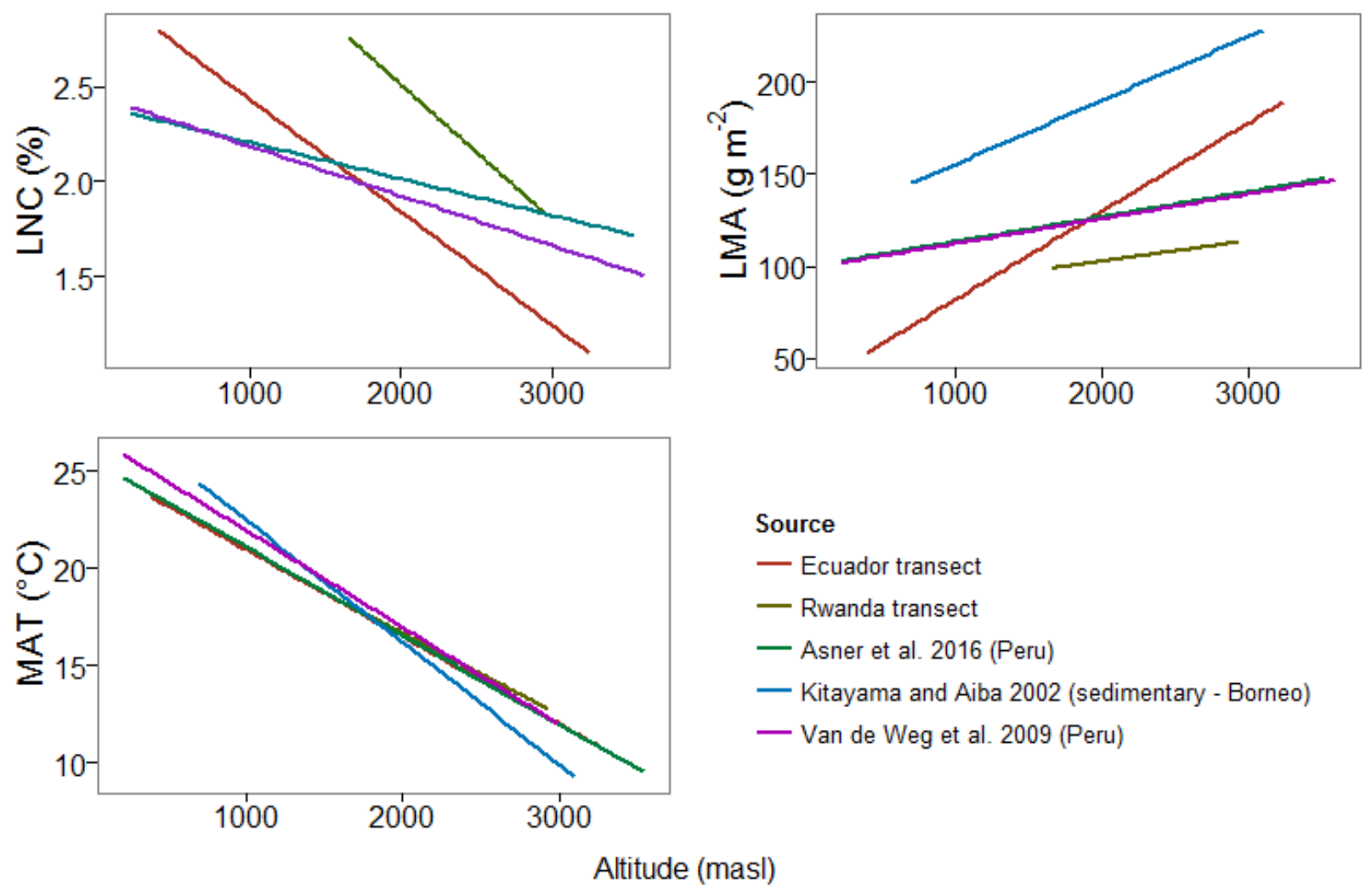

17

Fig. S3.

19 Trends in community-level leaf nitrogen content (LNC), leaf mass area (LMA) and mean annual

20 temperature (MAT) on different transects (as described in Asner et al., 2016; Kitayama \& Aiba,

21 2002; Van de Weg et al., 2009) compared to our transects in Ecuador and Rwanda. Figure was

22 obtained by using reported linear regression parameters, and by applying simple linear regression

23 lines to this study's data. 
25 Coordinates, elevation and cluster membership of the different plots on both transects

26

\begin{tabular}{|c|c|c|c|c|c|c|c|c|c|}
\hline \multirow[b]{2}{*}{ Plot } & \multirow[b]{2}{*}{ Cluster } & \multicolumn{3}{|c|}{ Ecuador } & \multirow[b]{2}{*}{ Plot } & \multirow[b]{2}{*}{ Cluster } & \multicolumn{3}{|c|}{ Rwanda } \\
\hline & & Latitude & Longitude & Elevation & & & Latitude & Longitude & Elevation \\
\hline 1 & 1 & $00^{\circ} 08^{\prime} 50.58^{\prime \prime} \mathrm{N}$ & $079^{\circ} 08^{\prime} 37.03^{\prime \prime}$ W & 406 & 1 & 4 & $02^{\circ} 26^{\prime} 47.28^{\prime \prime} \mathrm{S}$ & $029^{\circ} 15^{\prime} 09.96^{\prime \prime} \mathrm{E}$ & 2879 \\
\hline 2 & 1 & $00^{\circ} 08^{\prime} 45.79^{\prime \prime} \mathrm{N}$ & $079^{\circ} 08^{\prime} 34.45^{\prime \prime} \mathrm{W}$ & 420 & 2 & 4 & $02^{\circ} 26^{\prime} 50.93^{\prime \prime} \mathrm{S}$ & $029^{\circ} 15^{\prime} 07.55^{\prime \prime} \mathrm{E}$ & 2875 \\
\hline 3 & 1 & $00^{\circ} 08^{\prime} 50.31^{\prime \prime} \mathrm{N}$ & $079^{\circ} 08^{\prime} 31.87^{\prime \prime} \mathrm{W}$ & 404 & 3 & 4 & $02^{\circ} 26^{\prime} 25.68^{\prime \prime} \mathrm{S}$ & $029^{\circ} 15^{\prime} 00.96^{\prime \prime} \mathrm{E}$ & 2937 \\
\hline 4 & 1 & $00^{\circ} 08^{\prime} 50.49^{\prime \prime} \mathrm{N}$ & $079^{\circ} 08^{\prime} 33.30^{\prime \prime} \mathrm{W}$ & 410 & 4 & 4 & $02^{\circ} 27^{\prime} 09.89^{\prime \prime} \mathrm{S}$ & $029^{\circ} 14^{\prime} 57.36^{\prime \prime} \mathrm{E}$ & 2767 \\
\hline 5 & 1 & $00^{\circ} 08^{\prime} 50.27^{\prime \prime} \mathrm{N}$ & $079^{\circ} 08^{\prime} 35.04^{\prime \prime}$ W & 394 & 5 & 4 & $02^{\circ} 27^{\prime} 12.42^{\prime \prime} \mathrm{S}$ & $029^{\circ} 14^{\prime} 57.29^{\prime \prime} \mathrm{E}$ & 2761 \\
\hline 6 & 2 & $00^{\circ} 02^{\prime} 10.16^{\prime \prime} \mathrm{N}$ & $078^{\circ} 52^{\prime} 00.04^{\prime \prime} \mathrm{W}$ & 1098 & 6 & 2 & $02^{\circ} 28^{\prime} 52.79^{\prime \prime} \mathrm{S}$ & $029^{\circ} 11^{\prime} 04.08^{\prime \prime} \mathrm{E}$ & 2293 \\
\hline 7 & 2 & $00^{\circ} 02^{\prime} 08.27^{\prime \prime} \mathrm{N}$ & $078^{\circ} 51^{\prime} 59.52^{\prime \prime} \mathrm{W}$ & 1055 & 7 & 2 & $02^{\circ} 28^{\prime} 41.45^{\prime \prime} \mathrm{S}$ & $029^{\circ} 10^{\prime} 53.51^{\prime \prime} \mathrm{E}$ & 2240 \\
\hline 8 & 2 & $00^{\circ} 02^{\prime} 06.95^{\prime \prime} \mathrm{N}$ & $078^{\circ} 51^{\prime} 59.51^{\prime \prime} \mathrm{W}$ & 1077 & 8 & 1 & $02^{\circ} 27^{\prime} 33.59^{\prime \prime} \mathrm{S}$ & $029^{\circ} 12^{\prime} 02.52^{\prime \prime} \mathrm{E}$ & 1745 \\
\hline 9 & 2 & $00^{\circ} 02^{\prime} 11.07^{\prime \prime} \mathrm{N}$ & $078^{\circ} 52^{\prime} 02.51^{\prime \prime} \mathrm{W}$ & 1041 & 9 & 1 & $02^{\circ} 34^{\prime} 16.07^{\prime \prime} \mathrm{S}$ & $029^{\circ} 13^{\prime} 14.40^{\prime \prime} \mathrm{E}$ & 1835 \\
\hline 10 & 3 & $00^{\circ} 05^{\prime} 36.80^{\prime \prime} \mathrm{N}$ & $078^{\circ} 37^{\prime} 17.64^{\prime \prime} \mathrm{W}$ & 1953 & 10 & 1 & $02^{\circ} 34^{\prime} 15.35^{\prime \prime} \mathrm{S}$ & $029^{\circ} 13^{\prime} 04.25^{\prime \prime} \mathrm{E}$ & 1799 \\
\hline 11 & 3 & $00^{\circ} 05^{\prime} 45.48^{\prime \prime} \mathrm{N}$ & $078^{\circ} 37^{\prime} 19.25^{\prime \prime} \mathrm{W}$ & 1893 & 11 & 1 & $02^{\circ} 34^{\prime} 15.12^{\prime \prime} \mathrm{S}$ & $029^{\circ} 12^{\prime} 29.88^{\prime \prime} \mathrm{E}$ & 1760 \\
\hline 12 & 3 & $00^{\circ} 05^{\prime} 48.54^{\prime \prime} \mathrm{N}$ & $078^{\circ} 37^{\prime} 22.21^{\prime \prime} \mathrm{W}$ & 1873 & 12 & 1 & $02^{\circ} 34^{\prime} 21.47^{\prime \prime} \mathrm{S}$ & $029^{\circ} 12^{\prime} 01.91^{\prime \prime} \mathrm{E}$ & 1659 \\
\hline 13 & 3 & $00^{\circ} 06^{\prime} 06.87^{\prime \prime} \mathrm{N}$ & $078^{\circ} 37^{\prime} 40.73^{\prime \prime} \mathrm{W}$ & 1764 & 13 & 2 & $02^{\circ} 28^{\prime} 23.51^{\prime \prime} \mathrm{S}$ & $029^{\circ} 12^{\prime} 21.42^{\prime \prime} \mathrm{E}$ & 2141 \\
\hline 14 & 4 & $00^{\circ} 26^{\prime} 58.63^{\prime \prime} \mathrm{N}$ & $078^{\circ} 28^{\prime} 13.58^{\prime \prime} \mathrm{W}$ & 3214 & 14 & 2 & $02^{\circ} 28^{\prime} 13.91^{\prime \prime} \mathrm{S}$ & $029^{\circ} 12^{\prime} 29.69^{\prime \prime} \mathrm{E}$ & 2158 \\
\hline 15 & 4 & $00^{\circ} 26^{\prime} 59.20^{\prime \prime} \mathrm{N}$ & $078^{\circ} 28^{\prime} 14.94^{\prime \prime} \mathrm{W}$ & 3222 & 15 & 3 & $02^{\circ} 28^{\prime} 10.44^{\prime \prime} \mathrm{S}$ & $029^{\circ} 14^{\prime} 25.68^{\prime \prime} \mathrm{E}$ & 2557 \\
\hline 16 & 4 & $00^{\circ} 26^{\prime} 58.11^{\prime \prime} \mathrm{N}$ & $078^{\circ} 28^{\prime} 12.35^{\prime \prime} \mathrm{W}$ & 3191 & 16 & 3 & $02^{\circ} 28^{\prime} 11.09^{\prime \prime} \mathrm{S}$ & $029^{\circ} 14^{\prime} 18.17^{\prime \prime} \mathrm{E}$ & 2523 \\
\hline \multirow[t]{4}{*}{17} & 4 & $00^{\circ} 27^{\prime} 04.12^{\prime \prime} \mathrm{N}$ & $078^{\circ} 28^{\prime} 18.17^{\prime \prime} W$ & 3241 & 17 & 2 & $02^{\circ} 29^{\prime} 10.61^{\prime \prime} \mathrm{S}$ & $029^{\circ} 09^{\prime} 33.90^{\prime \prime} \mathrm{E}$ & 2167 \\
\hline & & & & & 18 & 3 & $02^{\circ} 28^{\prime} 43.97^{\prime \prime} \mathrm{S}$ & $029^{\circ} 12^{\prime} 07.85^{\prime \prime} \mathrm{E}$ & 2456 \\
\hline & & & & & 19 & 3 & $02^{\circ} 29^{\prime} 10.61^{\prime \prime} \mathrm{S}$ & $029^{\circ} 11^{\prime} 55.74^{\prime \prime} \mathrm{E}$ & 2500 \\
\hline & & & & & 20 & 3 & $02^{\circ} 29^{\prime} 14.16^{\prime \prime} \mathrm{S}$ & $029^{\circ} 11^{\prime} 42.83^{\prime \prime} \mathrm{E}$ & 2522 \\
\hline
\end{tabular}


29 Summary of the plot-level (mean and standard deviation) leaf nitrogen content (LNC), leaf phosphorus content (LPC), specific leaf area

30 (SLA), and leaf C:N, C:P, N:P and $\delta 15 \mathrm{~N}$, along with the total percentage of the basal area (BA) sampled per plot of the Ecuador transect.

\begin{tabular}{|c|c|c|c|c|c|c|c|c|c|c|}
\hline Transect & Plot & Altitude & SLA $\left(\mathrm{cm}^{2} \mathrm{~g}^{-1}\right)$ & LNC (\%) & $\operatorname{LPC}(\%)$ & $\mathrm{C}: \mathrm{N}$ & $\mathbf{C : P}$ & $\mathbf{N}: \mathbf{P}$ & $\delta^{15} \mathbf{N}(\%)$ & $\begin{array}{c}\text { Percentage } \\
\text { of BA } \\
\text { sampled } \\
\end{array}$ \\
\hline Ecuador & 1 & 404 & $149.74 \pm 66.55$ & $2.61 \pm 0.48$ & $0.13 \pm 0.03$ & $17.43 \pm 2.88$ & $359.7 \pm 65.25$ & $20.89 \pm 3.06$ & $2.7 \pm 1.5$ & $77 \%$ \\
\hline Ecuador & 2 & 420 & $136.43 \pm 42.31$ & $3.02 \pm 0.83$ & $0.15 \pm 0.05$ & $15.45 \pm 3.95$ & $320.39 \pm 107.88$ & $21.06 \pm 2.52$ & $3.24 \pm 1.4$ & $73 \%$ \\
\hline Ecuador & 3 & 404 & $148.83 \pm 54.33$ & $2.62 \pm 0.57$ & $0.13 \pm 0.03$ & $17.69 \pm 3.11$ & $356.46 \pm 76.83$ & $20.62 \pm 3.72$ & $2.66 \pm 0.79$ & $83 \%$ \\
\hline Ecuador & 4 & 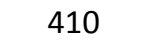 & $146.62 \pm 57.34$ & $2.61 \pm 0.59$ & $0.13 \pm 0.03$ & $17.64 \pm 3.26$ & $364.41 \pm 76.66$ & $20.92 \pm 3.03$ & $2.74 \pm 0.96$ & $66 \%$ \\
\hline Ecuador & 5 & 394 & $146.04 \pm 68.13$ & $2.57 \pm 0.58$ & $0.12 \pm 0.03$ & $17.73 \pm 3.15$ & $371.72 \pm 67.92$ & $21.32 \pm 3.26$ & $2.54 \pm 0.86$ & $61 \%$ \\
\hline Ecuador & 6 & 1098 & $142.58 \pm 35.07$ & $2.31 \pm 0.52$ & $0.14 \pm 0.04$ & $20.6 \pm 4.23$ & $369.76 \pm 137.5$ & $18.57 \pm 6.64$ & $-0.25 \pm 1.4$ & $30 \%$ \\
\hline Ecuador & 7 & 1055 & $164.48 \pm 46.3$ & $2.68 \pm 0.7$ & $0.15 \pm 0.06$ & $17.68 \pm 4.64$ & $320.68 \pm 120.44$ & $18.52 \pm 5.2$ & $1.12 \pm 1.87$ & $55 \%$ \\
\hline Ecuador & 8 & 1077 & $162.65 \pm 34.15$ & $2.73 \pm 0.57$ & $0.15 \pm 0.03$ & $17.22 \pm 3.61$ & $304.51 \pm 55.54$ & $18.12 \pm 2.35$ & $1.5 \pm 1.09$ & $55 \%$ \\
\hline Ecuador & 9 & 1041 & $135.56 \pm 45.96$ & $2.15 \pm 0.54$ & $0.14 \pm 0.05$ & $20.36 \pm 3.17$ & $332.95 \pm 72.91$ & $16.41 \pm 2.91$ & $0.77 \pm 1.67$ & $44 \%$ \\
\hline Ecuador & 10 & 1953 & $115.76 \pm 25.38$ & $2.08 \pm 0.43$ & $0.13 \pm 0.01$ & $22.48 \pm 5.34$ & $341.55 \pm 43.48$ & $15.78 \pm 2.54$ & $0.22 \pm 0.81$ & $64 \%$ \\
\hline Ecuador & 11 & 1893 & $123.92 \pm 26.67$ & $1.98 \pm 0.57$ & $0.12 \pm 0.03$ & $23.74 \pm 7.24$ & $365.68 \pm 95$ & $16.02 \pm 2.5$ & $-0.04 \pm 1.1$ & $83 \%$ \\
\hline Ecuador & 12 & 1873 & $113.68 \pm 29.9$ & $2.28 \pm 0.38$ & $0.15 \pm 0.04$ & $20.51 \pm 3.75$ & $323.25 \pm 80.78$ & $16.44 \pm 4.47$ & $0.18 \pm 0.73$ & $76 \%$ \\
\hline Ecuador & 13 & 1764 & $113.57 \pm 18.51$ & $1.99 \pm 0.38$ & $0.13 \pm 0.01$ & $23.28 \pm 4.46$ & $360.03 \pm 37.76$ & $15.67 \pm 2.39$ & $0.12 \pm 0.52$ & $72 \%$ \\
\hline Ecuador & 14 & 3214 & $47.95 \pm 26.12$ & $0.97 \pm 0.36$ & $0.06 \pm 0.01$ & $50.96 \pm 13.85$ & $772.64 \pm 115.72$ & $15.56 \pm 3.55$ & $-5.07 \pm 1.94$ & $85 \%$ \\
\hline Ecuador & 15 & 3222 & $49.29 \pm 24.14$ & $1.02 \pm 0.47$ & $0.06 \pm 0.01$ & $49.43 \pm 15.54$ & $756.2 \pm 138.32$ & $15.86 \pm 3.99$ & $-4.83 \pm 2.3$ & $95 \%$ \\
\hline Ecuador & 16 & 3191 & $46.88 \pm 18.44$ & $0.98 \pm 0.43$ & $0.06 \pm 0.01$ & $51.07 \pm 14.52$ & $770.36 \pm 141.15$ & $15.46 \pm 3.23$ & $-5.05 \pm 2.32$ & $91 \%$ \\
\hline Ecuador & 17 & 3241 & $59.27 \pm 30.31$ & $1.16 \pm 0.38$ & $0.07 \pm 0.01$ & $44.08 \pm 14.2$ & $697.04 \pm 151.06$ & $16.47 \pm 2.8$ & $-4.23 \pm 1.84$ & $95 \%$ \\
\hline
\end{tabular}


Table S2b.

33 Summary of the plot-level (mean and standard deviation) leaf nitrogen content (LNC), leaf phosphorus content (LPC), specific leaf area

34 (SLA) and leaf C:N, C:P, N:P and $\delta 15 \mathrm{~N}$, along with the total percentage of the basal area (BA) sampled per plot of the Rwanda transect.

\begin{tabular}{|c|c|c|c|c|c|c|c|c|c|c|}
\hline Transect & Plot & Altitude & SLA $\left(\mathbf{c m}^{2} \mathbf{g}^{-1}\right)$ & LNC (\%) & LPC (\%) & $\mathrm{C}: \mathrm{N}$ & $\mathrm{C}: \mathrm{P}$ & $\mathrm{N}: P$ & $\delta^{15} \mathbf{N}(\%)$ & $\begin{array}{c}\text { Percentage } \\
\text { of BA } \\
\text { sampled } \\
\end{array}$ \\
\hline Rwanda & 1 & 2879 & $42.04 \pm 28.52$ & $1.53 \pm 0.94$ & $0.09 \pm 0.04$ & $34.58 \pm 13.74$ & $564.61 \pm 171.59$ & $16.81 \pm 2.85$ & $-0.12 \pm 1.46$ & $90 \%$ \\
\hline Rwanda & 2 & 2875 & $45.83 \pm 23.33$ & $1.83 \pm 1.03$ & $0.1 \pm 0.05$ & $30.78 \pm 14.2$ & $508.9 \pm 191.33$ & $17.16 \pm 2.3$ & $0.52 \pm 1.89$ & $95 \%$ \\
\hline Rwanda & 3 & 2937 & $35.53 \pm 26.65$ & $1.33 \pm 1.09$ & $0.08 \pm 0.05$ & $37.72 \pm 15.34$ & $598.75 \pm 202.9$ & $16.09 \pm 2.42$ & $-0.2 \pm 1.6$ & $99 \%$ \\
\hline Rwanda & 4 & 2767 & $60.76 \pm 31.92$ & $2.09 \pm 0.93$ & $0.11 \pm 0.04$ & $27.67 \pm 11.72$ & $483.82 \pm 147.81$ & $19.26 \pm 5.05$ & $0.8 \pm 1.73$ & $91 \%$ \\
\hline Rwanda & 5 & 2761 & $60.19 \pm 32.62$ & $2.09 \pm 0.98$ & $0.11 \pm 0.04$ & $27.95 \pm 12.02$ & $488.41 \pm 149.64$ & $19.55 \pm 5.66$ & $0.68 \pm 1.59$ & $97 \%$ \\
\hline Rwanda & 6 & 2293 & $99.03 \pm 21.26$ & $2.54 \pm 0.49$ & $0.12 \pm 0.03$ & $19.85 \pm 3.8$ & $398.62 \pm 86.35$ & $21.01 \pm 3.94$ & $3.2 \pm 1.85$ & $86 \%$ \\
\hline Rwanda & 7 & 2240 & $112.49 \pm 25.8$ & $2.49 \pm 0.53$ & $0.13 \pm 0.02$ & $20.88 \pm 4.5$ & $380.75 \pm 78.3$ & $19.57 \pm 3.17$ & $4.07 \pm 1.9$ & $94 \%$ \\
\hline Rwanda & 8 & 1745 & $110.11 \pm 25.21$ & $2.59 \pm 0.66$ & $0.13 \pm 0.02$ & $20.59 \pm 4.03$ & $385.18 \pm 73.06$ & $20.44 \pm 3.37$ & $3.52 \pm 1.94$ & $99 \%$ \\
\hline Rwanda & 9 & 1835 & $106.3 \pm 32.8$ & $2.68 \pm 0.74$ & $0.13 \pm 0.04$ & $19.81 \pm 4.91$ & $384.09 \pm 96.91$ & $20.83 \pm 3.7$ & $3.8 \pm 1.83$ & $97 \%$ \\
\hline Rwanda & 10 & 1799 & $106.11 \pm 30.81$ & $2.62 \pm 0.72$ & $0.13 \pm 0.05$ & $20.19 \pm 5.37$ & $388.53 \pm 115.94$ & $20.4 \pm 5.11$ & $4.23 \pm 1.98$ & $92 \%$ \\
\hline Rwanda & 11 & 1760 & $111.25 \pm 21.64$ & $2.6 \pm 0.63$ & $0.12 \pm 0.02$ & $20.4 \pm 4.39$ & $388.3 \pm 62.55$ & $20.89 \pm 3.51$ & $3.97 \pm 1.48$ & $100 \%$ \\
\hline Rwanda & 12 & 1659 & $104.19 \pm 39.07$ & $2.39 \pm 0.71$ & $0.12 \pm 0.04$ & $21.88 \pm 4.92$ & $404.88 \pm 97.93$ & $19.63 \pm 3.97$ & $3.51 \pm 2.27$ & $100 \%$ \\
\hline Rwanda & 13 & 2141 & $102.67 \pm 21.38$ & $2.61 \pm 0.56$ & $0.12 \pm 0.03$ & $19.39 \pm 4.36$ & $413.08 \pm 102.42$ & $22.01 \pm 4.45$ & $4.01 \pm 1.99$ & $97 \%$ \\
\hline Rwanda & 14 & 2158 & $105.99 \pm 22.76$ & $2.55 \pm 0.39$ & $0.11 \pm 0.02$ & $20.85 \pm 3.29$ & $439.03 \pm 66.3$ & $23.06 \pm 3.52$ & $3.31 \pm 1.42$ & $100 \%$ \\
\hline Rwanda & 15 & 2557 & $98.84 \pm 24.87$ & $2.5 \pm 0.73$ & $0.11 \pm 0.02$ & $22.43 \pm 4.52$ & $433.44 \pm 65.02$ & $22.89 \pm 6.92$ & $1.01 \pm 0.88$ & $99 \%$ \\
\hline Rwanda & 16 & 2523 & $100.12 \pm 27.99$ & $2.38 \pm 0.73$ & $0.11 \pm 0.02$ & $23.16 \pm 5.33$ & $440.96 \pm 61.48$ & $22.02 \pm 6.44$ & $0.94 \pm 0.8$ & $100 \%$ \\
\hline Rwanda & 17 & 2167 & $89.7 \pm 30.72$ & $2.18 \pm 0.63$ & $0.11 \pm 0.03$ & $24.28 \pm 7.86$ & $448.71 \pm 101.96$ & $19.45 \pm 2.82$ & $1.93 \pm 1.83$ & $97 \%$ \\
\hline Rwanda & 18 & 2456 & $94.81 \pm 32.58$ & $2.04 \pm 0.46$ & $0.11 \pm 0.02$ & $25.36 \pm 5.17$ & $452.75 \pm 75.93$ & $19.01 \pm 2.84$ & $0.94 \pm 0.9$ & $99 \%$ \\
\hline Rwanda & 19 & 2500 & $89.29 \pm 32.47$ & $2.24 \pm 0.43$ & $0.12 \pm 0.02$ & $21.6 \pm 3.85$ & $386.74 \pm 73.89$ & $18.15 \pm 1.34$ & $0.98 \pm 1.27$ & $93 \%$ \\
\hline Rwanda & 20 & 2522 & $86.38 \pm 23.57$ & $2.2 \pm 0.55$ & $0.12 \pm 0.03$ & $23.02 \pm 5.39$ & $410.66 \pm 91.2$ & $18.45 \pm 0.81$ & $1.36 \pm 1.13$ & $98 \%$ \\
\hline
\end{tabular}


41 interaction term.

\begin{tabular}{|c|c|c|c|c|c|}
\hline Response & Effect & Estimate & Standard Error & P-value & $\triangle \mathrm{AIC}$ \\
\hline \multirow[t]{4}{*}{ LNC (\%) } & Ecuador intercept & 3.010 & 0.145 & $<0.001$ & 1.10 \\
\hline & Rwanda intercept & 3.930 & 0.466 & 0.114 & \\
\hline & Altitude & -0.572 & 0.000 & 0.001 & \\
\hline & Altitude:Transect & -0.140 & 0.000 & 0.525 & \\
\hline \multirow[t]{4}{*}{$\operatorname{SLA}\left(\mathrm{cm}^{2} \mathrm{~g}^{-1}\right)$} & Ecuador intercept & 171.377 & 15.534 & $<0.001$ & 1.16 \\
\hline & Rwanda intercept & 199.016 & 44.535 & 0.552 & \\
\hline & Altitude & -33.849 & 0.008 & 0.009 & \\
\hline & Altitude:Transect & -13.755 & 0.019 & 0.496 & \\
\hline \multirow[t]{4}{*}{$\mathrm{N}: \mathrm{C}$} & Ecuador intercept & 0.066 & 0.004 & $<0.001$ & 1.94 \\
\hline & Rwanda intercept & 0.075 & 0.011 & 0.472 & \\
\hline & Altitude & -0.013 & 0.000 & 0.002 & \\
\hline & Altitude:Transect & -0.000 & 0.000 & 0.930 & \\
\hline \multirow[t]{4}{*}{ LPC (\%) } & Ecuador intercept & 0.157 & 0.014 & $<0.001$ & 2.00 \\
\hline & Rwanda intercept & 0.170 & 0.042 & 0.763 & \\
\hline & Altitude & -0.024 & 0.000 & 0.024 & \\
\hline & Altitude:Transect & 0.000 & 0.000 & 0.985 & \\
\hline \multirow[t]{4}{*}{$N: P$} & Ecuador intercept & 20.440 & 1.215 & $<0.001$ & 1.60 \\
\hline & Rwanda intercept & 25.885 & 3.842 & 0.218 & \\
\hline & Altitude & -1.686 & 0.001 & 0.051 & \\
\hline & Altitude:Transect & -0.913 & 0.002 & 0.607 & \\
\hline
\end{tabular}


44 NASA/METI: The Advanced Spaceborne Thermal Emission and Reflection Radiometer (ASTER) Global Digital 45 Elevation Model (GDEM) version 2, [online] Available from: http://earthexplorer.usgs.gov/, 2011.

46

47

48

56

57

58

59

60

61

62

63

Asner, G. P., Martin, R. E., Anderson, C. B., Kryston, K., Vaughn, N., Knapp, D. E., Bentley, L. P., Shenkin, A., Salinas, N., Sinca, F., Tupayachi, R., Sandra, D., Huaypar, K. Q., Pillco, M. M., Delis, F., Alvarez, C., Malhi, Y., Quispe Huaypar, K., Montoya Pillco, M., Ccori Álvarez, F. D., Díaz, S., Enquist, B., Malhi, Y., Sandra, D., Huaypar, K. Q., Pillco, M. M., Delis, F., Alvarez, C. and Malhi, Y.: Scale dependence of canopy trait distributions along a tropical forest elevation gradient, New Phytol., 214, 973-988, doi:10.1111/nph.14068, 2016.

Kitayama, K. and Aiba, S.: Ecosystem structure and productivity of tropical rain forests along altitudinal gradients with contrasting soil phosphorus pools on Mount Kinabalu , Borneo, J. Ecol., 90, 37-51, doi:10.1046/j.00220477.2001.00634.x, 2002.

Van de Weg, M. J., Meir, P., Grace, J., Atkin, O. K..: Altitudinal variation in leaf mass per unit area, leaf tissue density and foliar nitrogen and phosphorus content along an Amazon-Andes gradient in Peru, Plant Ecol. Divers., 2, 243-254, doi:10.1080/17550870903518045, 2009.

Nakagawa, S. and Schielzeth, H.: A general and simple method for obtaining $\mathrm{R}^{2}$ from generalized linear mixed-effects models, edited by R. B. O’Hara, Methods Ecol. Evol., 4, 133-142, doi:10.1111/j.2041-210x.2012.00261.x, 2013. 